\title{
Análisis del voto de sensación térmica percibida para espacios exteriores, en el verano de la zona metropolitana de Tijuana, México
}

\section{Analysis of the vote of perceived thermal sensation for outdoor spaces, in the summer of the metropolitan area of Tijuana, Mexico}

\author{
Miguel Isaac, Sahagun Valenzuela \\ María de los Ángeles, Zarate López ${ }^{2}$ \\ Juan Antonio, Pitones Rubio ${ }^{3}$ \\ Alberto, Almejo Ornelas ${ }^{4}$ \\ ${ }^{1-4}$ Universidad Autónoma de Baja California, Tecate, México, \\ 1isahagun@uabc.edu.mx / https://orcid.org/0000-0001-6363-0884 \\ 2maria.zarate@uabc.edu.mx / https://orcid.org/0000-0002-1554-5439 \\ 3juan.antonio.pitones.rubio@uabc.edu.mx / https://orcid.org/0000-0002-9897-1846 \\ 4alberto.almejo@uabc.edu.mx / https://orcid.org/0000-0002-0461-9383
}

Recibido el 12 de enero de 2021, aprobado el 27 de diciembre de 2021

RESUMEN | El tipo de condiciones meteorologías que se presentan en un espacio exterior, provocan un tipo de uso del espacio, así como la permanencia del usuario en el mismo, por la sensación de confort provocada por las condiciones climáticas, esto a diferencia de los espacios interiores donde se puede controlar sistemas de enfriamiento o calefacción, lo cual protege al usuario de las condiciones climatológicas.

Para la evaluación del confort en espacios exteriores de la ciudad de Tijuana, Baja California, se seleccionó un caso de estudio, en el cual se llevó a cabo la evaluación de sensación térmica percibida, por medio de levantamiento de encuestas de percepción, donde se utilizaron criterios de vestimenta, genero, actividad, edad, permanencia, uso adecuado del sitio, al mismo tiempo que se realizaba la encuesta se tomaba la temperatura de bulbo seco, humedad relativa, el peso y la altura del usuario, se recopilo la información de sensación térmica basado en la norma ISO 7730-2005.

Se realizó el análisis de los datos estadísticos, y se pudo observar cual es la temperatura de confort, con la cual se podrán diseñar espacios apropiados y adecuaciones a espacios existentes ya en uso, con lo que se aprovecharán los espacios exteriores durante las temperaturas críticas.

PALABRAS CLAVE | Confort Térmico, espacio público, sensación térmica, clima seco, espacio exterior

ABSTRACT | The type of meteorological conditions that occur in outer space, cause a kind of 
use of space, as well as the permanence of the user in it, for the feeling of comfort caused by weather conditions, this unlike interior spaces where you can control cooling or heating systems, which protects the user from weather conditions.

For the evaluation of comfort in outdoor spaces in the city of Tijuana, Baja California, a case study was selected, in which the evaluation of perceived thermal sensation was carried out, through the application of perception surveys, where they were used clothing criteria, gender, activity, age, permanence, proper use of the site, at the same time as the survey was carried out, the dry bulb temperature, relative humidity, the weight and height of the user, the thermal sensation information was collected based on the ISO 7730-2005 standard.

Statistical data analysis was performed, and you could see what is the comfort temperature, with which appropriate spaces and adaptations to existing spaces can be designed, with what outside spaces will be utilized during critical temperatures.

KEYWORD | Thermal comfort, public space, thermal sensation, dry weather, outdoor space

\section{Introducción}

Los espacios públicos y las condiciones climáticas en los espacios exteriores condicionan el uso y la permanencia del usuario en el lugar, al contrario que en los espacios interiores los cuales pueden ser controlados, pudiendo alterar las condiciones de vida de los ocupantes mejorando las condiciones climáticas que puedan afectarles acercándolos a la zona de confort. Estos cambios al hábitat de los ocupantes del espacio se deben a las mejoras que pueden ser añadidas durante el proceso de diseño de la edificación, controlando los aspectos climáticos y adecuando el lugar para llegar a un espacio confortable adecuado para el ocupante.

Para buscar evaluar la comodidad o confort térmico de los espacios públicos en la zona metropolitana de Tijuana, Baja California, México, y considerar su habitabilidad transitoria, se escogió el caso para su investigación y se evaluó la sensación de calor percibida, por medio de un instrumento diseñado para tal fin, aplicado durante el verano de 2019. De acuerdo a como se encontraba el usuario y que actividad realizada se incluyeron aspectos como; el tipo de ropa, genero, edad, y preguntas sobre la satisfacción, durabilidad, al mismo tiempo que se realizaba la encuesta se tomaba la temperatura de bulbo seco, humedad relativa, el peso y la altura del usuario, se recopilo la información de sensación térmica basado en la norma ISO 7730-2005.

Una vez analizados los datos recabados se puede obtener la temperatura de neutralidad, o zona de confort votada por le usuario para el espacio abierto, esta se puede utilizar para futuros diseños de espacios similares o adecuar los lugares públicos existentes en la zona metropolitana de Tijuana, así como otras ciudades con el mismo o similar tipo de clima a la ciudad analizada, promoviendo así la restauración de la convivencia y socialización en espacios públicos abiertos. Se propone una estimación de la temperatura de confort térmico del espacio exterior publico en el período cálido del clima semidesértico. Se realizaron 249 encuestas durante el verano de 2019. La temperatura de neutralidad y el rango de confort se calculan utilizando el método de correlación del valor medio y la dispersión estándar de los datos obtenidos. (Guzmán y Ochoa, 2014) 
Para obtener el diagnóstico de un espacio en el que se demuestre que un área se encuentra dentro de las zonas de confort o fuera de esta, así como la intervención sobre las estrategias de diseño de parques o espacio abiertos fue necesario utilizar las estrategias de métodos inductivos con investigaciones de campo y de variables climáticas, esto para mejorar el tiempo de utilización de los espacios por los usuarios.

\section{Confort térmico}

El ser humando siempre se ha esforzado por crear lugares con un ambiente térmicamente cómodo, esto se puede reflejar en las construcciones tradicionales que se encuentra a lo largo de todo el mundo, los cuales van desde la historia antigua, hasta el presente que vivimos. Hoy para crear un ambiente térmicamente cómodo todavía es uno de los parámetros mas importantes a ser considerados cuando se planifica y diseña una edificación moderna. (Chávez, 2002)

Para las personas en espacios exteriores, el confort térmico es uno de los factores que afectan las actividades al aire libre realizadas en las calles, plazas, parques infantiles y parques de la ciudad. El número e intensidad de estas actividades se ven afectados por el grado de malestar que experimentan los usuarios ante las condiciones climáticas de a estos espacios exteriores. Si no hay molestias térmicas de ningún tipo, la persona pensará que el ambiente es agradable. La primera condición de confort es la neutralidad térmica, lo que significa que uno no se siente ni demasiado caliente ni demasiado frío. (Guzmán, y Ochoa, 2014)

El confort térmico el cual es definido en la norma ISO 7730 (2005) como "Esa condición de mente en la que se expresa la satisfacción con el ambiente térmico" (p. 5). La cual es una definición que se adecuada la mayor parte de las personas y por lo tanto es aceptada, pero al mismo tiempo es una definición que tiene su dificultad por basarse en la subjetividad del individuo que la expresa, en la figura 1 se puede apreciar la complejidad de la evaluación del confort térmico, en las dos figuras se encuentras personas en una misma ciudad y zona de la misma, pero en situaciones muy distintas, pero ambas pueden estar en confort térmico a pesar de la diferencia. (Chávez, 2002).

Figura 2. Alumnos en confort

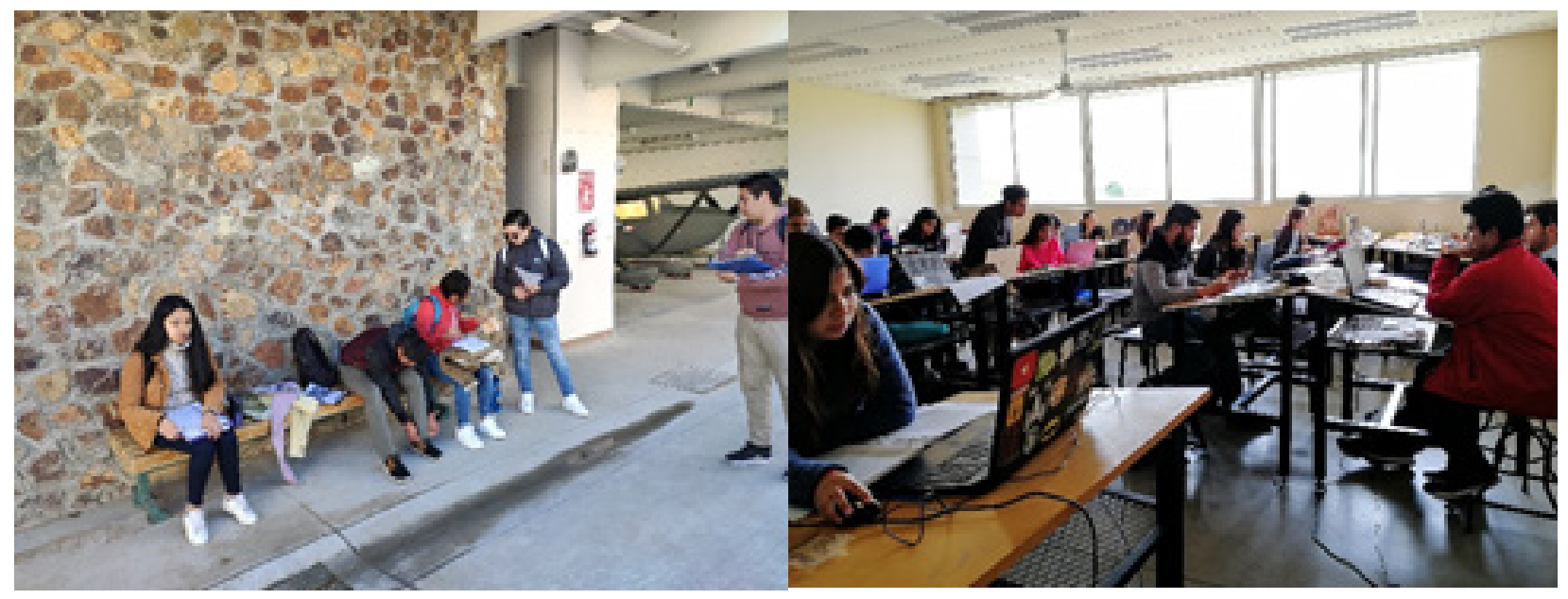

Nota. Alumnos en distintas situaciones y ambientes, pero ambos en confort 


\section{Metodología}

Los procesos para investigar el confort térmico en espacios públicos exteriores son relativamente nuevos y en ellos influyen variables que son distintas a las que se pueden apreciar en el confort para espacios interiores, al tener un control menor de lo que sucede en el espacio exterior, esto conlleva una mezcla de mayor complejidad entre las correlaciones entre los parámetros con una mayor varianza, ya sea por el poco control del comportamiento climático o de las actividades que puedan realizar los ocupantes del espacio en el lugar es por esto que los usuarios se enfrentan a los efectos de la adaptación en las condiciones del ambiente exterior. (Gómez et al., 2010)

La investigación se realiza mediante la aplicación de un instrumento donde el usuario plasma de manera subjetiva sus sensaciones en el momento, y al mismo tiempo se toman mediciones de temperatura de bulbo seco y humedad relativa, así como el peso de los encuestados y su altura corporal. Para el desarrollo del cuestionario se tomo como base las encuestas diseñadas por Bojórquez et al. (2010), por Nikolopolou (2004) y por Guzman (2014).

Durante el levantamiento de datos de campo se tuvo la participación de los usuarios del espacio exterior, dando sus repuestas al momento de aplicar el instrumento, el cual estaba dividido de varias secciones, primero los datos de ubicación y hora de aplicación, segundo el tipo de actividad realizada durante la aplicación de la encuesta, tercero los datos del usuario edad, peso altura, genero, después su sensación térmica y de humedad al momento de aplicar el instrumento, y después la permanecía del usuario en el espacio analizado.

En la cuarta parte del instrumento se consideran diversos aspectos de la adaptación psicológica y física, para comprender la evaluación de los diferentes parámetros climatológicos, la percepción de calor y el gusto de los usuarios, donde se les pidió a los usuarios en su sensación puntual, que emitieran una evaluación dentro de las opciones disponibles para el cuestionario, de acuerdo con la clasificación ISO 7730 (2005).

Durante el llenado de la quinta parte del instrumento, se escribieron los datos del monitoreo de campo realizado simultáneamente. Donde se anotaron los datos de temperatura de bulbo seco y humedad relativa, con las que se podrá conocer a las condiciones a las que los usuarios del espacio exterior estaban expuestos en el momento, todo esto con el objetivo de conocer las condiciones de los encuestados, donde se utilizaron data logger portátiles.

Para la selección de los instrumentos de medición se tomaron en cuenta tres factores los cuales son la precisión, disponibilidad del equipo y la facilidad de uso, la precisión del equipo elegidos es muy importante por lo que se buscó equipos con un error no mayor de $0.5^{\circ} \mathrm{C}$, el elegido fue de $0.1^{\circ} \mathrm{C}$

En cuanto a disponibilidad se buscó equipo comercial fácil de conseguir con las características necesarias de medición, se buscó también que sea de entrega rápida, se buscó que el equipo fuera fácil de utilizar, sin requerir una capacitación extensa, ya que el tiempo para realizar el experimento es limitado, una característica del equipo es que fuera fácil de conectar con una computadora para sincronizar la información fácilmente.

Para el monitoreo se utilizó equipo diseñado para medir temperatura por medio de termopares, con una confiabilidad de $\pm 0.1^{\circ} \mathrm{C}$, con un rango de medición de $-20^{\circ} \mathrm{C}$ a $70^{\circ} \mathrm{C}$, y un año de vida en la batería con 52000 mediciones (figura 2). 
Figura 2. Equipo de medición

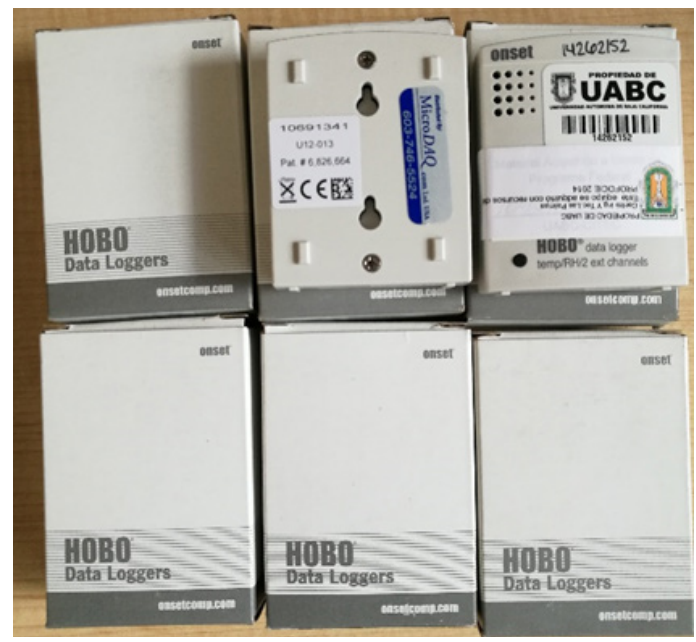

Nota. Equipo con el que se registró la temperatura y humedad relativa durante la aplicación de las encuestas

Para el análisis de los datos se utilizó el software de Microsoft Excel, donde se capturaron los datos recopilados durante el levantamiento de campo en papel, con lo que se realizo la base de datos, y a su vez se capturo los datos climáticos monitoreados durante el mismo levantamiento, en el programa se hizo un ordenamiento de los datos, se agruparon en rubros por tipos de sensaciones térmica percibida, se capturaron 43 variables distintas entra las cuales se incluyen la edad, el género, el peso, la altura, el tipo de vestimenta, el lugar del espacio donde se aplicó la encuesta, y el voto de sensación térmica percibida en el momento.

\section{Clima y localización}

Según Linacre y Geerts (1997) el clima se puede definir como la conjunción de las condiciones atmosféricas que son características de un sitio, el cual es definido por medio de los promedios de los parámetros meteorológicos y por la probabilidad de la ocurrencia de las condiciones que incluyen eventos climatológicos extremos, el factor temporal también afecta al clima en todas sus escalas, ya que el clima depende del movimiento del planeta, rotación y traslación, que dan la pauta para los cambios secuenciales climáticos.

Figura 3. Zona metropolitana de Tijuana

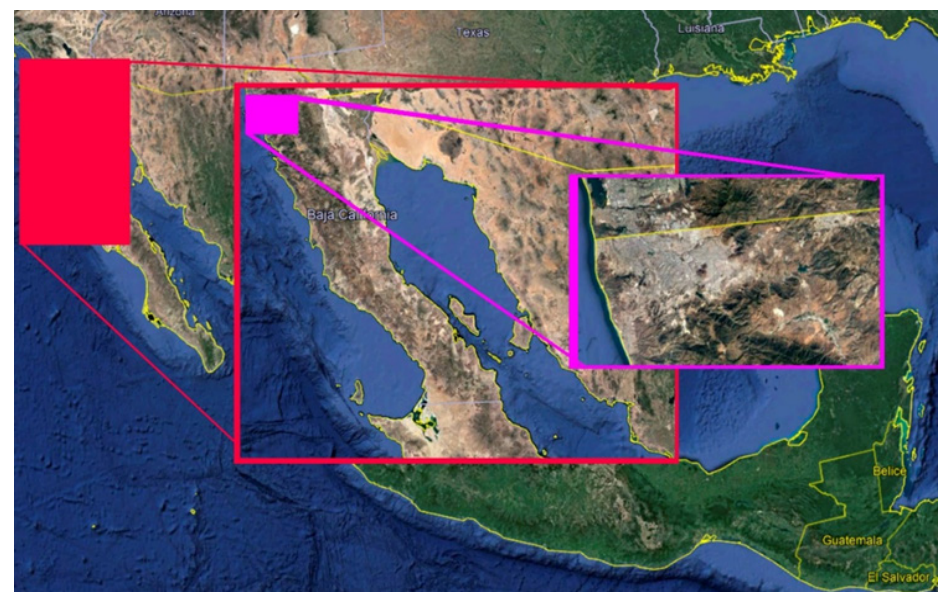

Nota. Vista satelital del área metropolitana de Tijuana y condado de San Diego 
El sitio de la aplicación de las encuestas se ubicó dentro de la zona metropolitana de Tijuana en la unidad de Valle de las Palmas que se encuentra ubicada en las coordenadas $32^{\circ} 25^{\prime} 59^{\prime \prime} \mathrm{N}, 116^{\circ}$ 40' 31" O (Figura 3). La Zona Metropolitana de Tijuana incluye los Municipios de Tijuana, Tecate y Playas de Rosarito la zona tiene colindancia al Este con el Municipio de Mexicali, Al Oeste con el Océano Pacifico, Al sur con el Municipio de Ensenada y Al Norte con el Condado de San Diego y al Noreste con el Condado de Imperial en California.

El espacio exterior elegido se encuentra al interior de los terrenos de la Universidad Autónoma de Baja California, unidad Valle de las Palmas, son espacios públicos y comunes que puede ser visitados tanto por los alumnos del plantel, como por los visitantes que lleguen a las instalaciones de la unidad figura 4.

Figura 4. Valle de las palmas

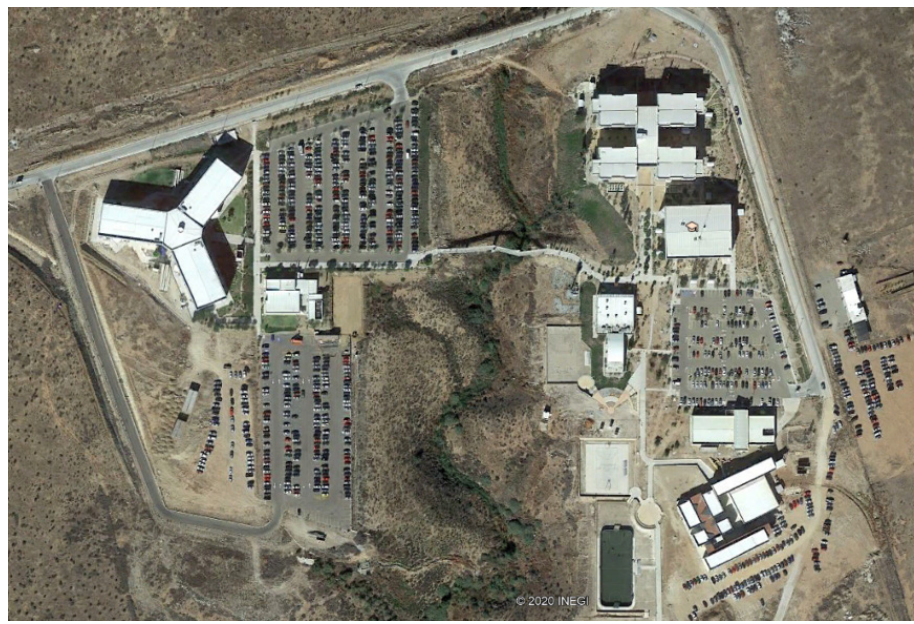

Nota. En la imagen se puede apreciar una vista aérea del campus de la UABC de Valle de las palmas, dentro de la zona metropolitana de Tijuana.

La unidad de valle de las palmas cuenta con una superficie de $240668 \mathrm{~m}^{2}$. Su ubicación dentro de la zona metropolitana de Tijuana, es al sureste y está rodeada al este y al sur del valle de las palmas de Tecate, Baja California, al norte por la colonia de valle de san pedro y villa el campo, y al oeste por la colonia ciudad sustentable valle de las palmas. Cuenta con grandes explanadas pavimentadas con concreto y áreas deportivas Figura 5.

Figura 5. Áreas comunes

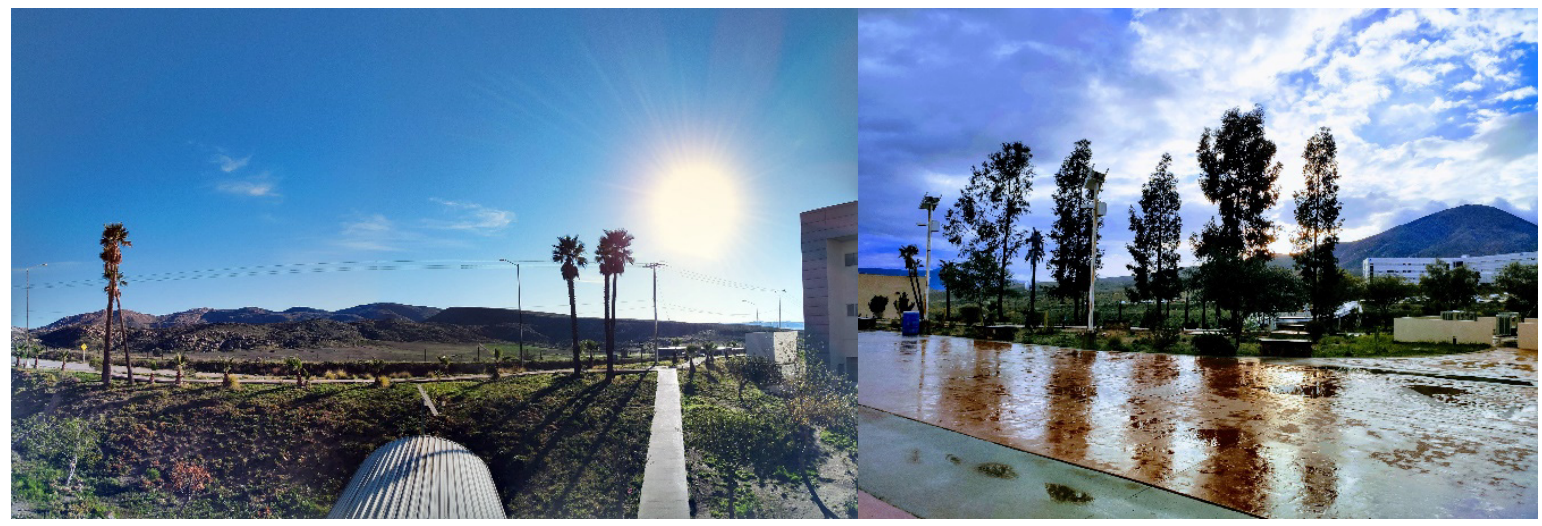

Nota. Áreas comunes de espacio exterior dentro de la unidad de valle de las palmas 


\section{Análisis y resultados}

Al realizar el monitoreo de variables meteorológicas simultáneamente a la aplicación del instrumento de evaluación subjetiva del usuario, fueron seleccionados de los datos utilizados el valor máximo de la temperatura del ambiente o Temp. Amb. Max, la cual registro un valor de $37.02^{\circ} \mathrm{C}$, así mismo se registró un valor mínimo para la temperatura del ambiente o Temp. Amb. Min. la cual fue $30.21^{\circ} \mathrm{C}$, dentro del mismo registro se pudo obtener los valores de humedad relativa máxima o H.R. Max, con un valor de $60 \%$ y también el valor mínimo de la humedad relativa la cual registro un $11 \%$ (Tabla. 1 ).

Tabla 1. Máximos, mínimos y promedios de variables

\begin{tabular}{llll}
\hline \multicolumn{4}{l}{ Unidad Valle de las Palmas } \\
\hline $\begin{array}{l}\text { Temp. Amb. } \\
\text { Max }{ }^{\circ} \mathrm{C}\end{array}$ & $\begin{array}{l}\text { Temp. Amb. } \\
\text { Min }{ }^{\circ} \mathrm{C}\end{array}$ & H.R. Max \% & H.R. Min \% \\
\hline 37.02 & 30.21 & 60 & 11 \\
\hline 33.62 & & 35.50 & \\
\hline
\end{tabular}

El instrumento fue aplicado en un total de 249 personas en el verano del año 2019, los cuales dieron su voto de sensación térmica percibida distribuido de la siguiente forma, el $30 \%$ de las respuestas fue por sentirse en un ambiente sin frio ni calor lo que representa estar en neutralidad o confortable, el $21 \%$ dio su voto por sentirse con algo de calor lo cual seria tener una leve incomodidad al ambiente percibido, y el 49\% de los votos fueron tener calor en el lugar lo que representa sentir incomodidad por el espacio con respecto al ambiente térmico (Figura 6).

Figura 6. Porcentajes de voto de sensación térmica



Nota. Porcentajes de votos recibidos durante la aplicación de la encuesta

Para el análisis de los datos se utilizó el Método de Medidas por Intervalo de Sensación Térmica o MIST, en el cual se establecieron rubros de la misma para calcular los valores promedio y la desviación estándar de todos los grupos formados. Realizando, una regresión lineal utilizando solo los valores medios de la muestra y no utilizando todos los valores, y cada rango es establecido sumando y restando la desviación estándar de la muestra una y dos veces (Bojórquez, G., et al, 2010)

Una vez aplicado el método MIST, fueron obtenidos los datos que pueden ser vistos en la tabla 
Tabla 2. Tn y rango reducido y extenso

\begin{tabular}{llllllll}
\hline DS & $\begin{array}{l}\text { Sensación } \\
\text { Térmica }\end{array}$ & Escala & TN-2DS & TN-1DS & TN & TN+1DS & TN+2DS \\
\hline 1.96 & $\begin{array}{l}\text { Ni frio ni } \\
\text { calor }\end{array}$ & 4 & 29.23 & 31.18 & 33.14 & 35.10 & 37.05 \\
\hline
\end{tabular}

Nota. Porcentajes de votos recibidos durante la aplicación de la encuesta

2, donde se pueden apreciar la Tn o Temperatura Neutra, así como el rango reducido y el rango extenso del confort térmico, con base en la ecuación de la recta de regresión lineal aplicada para los valores medios.

La temperatura de neutralidad o Tn para el espacio medido y analizado tiene un valor de $33.14^{\circ} \mathrm{C}$, con un rango reducido de confort térmico de $3.92^{\circ} \mathrm{C}, \mathrm{o} \pm 1.9^{\circ} \mathrm{C}$ a partir de la temperatura de neutralidad, y un rango extendido de confort térmico de $7.84^{\circ} \mathrm{C}, \mathrm{o} \pm 3.9^{\circ} \mathrm{C}$ a partir de la temperatura de neutralidad (Figura 7).

Tabla 7. Voto de sensación térmica por temperatura ambiente

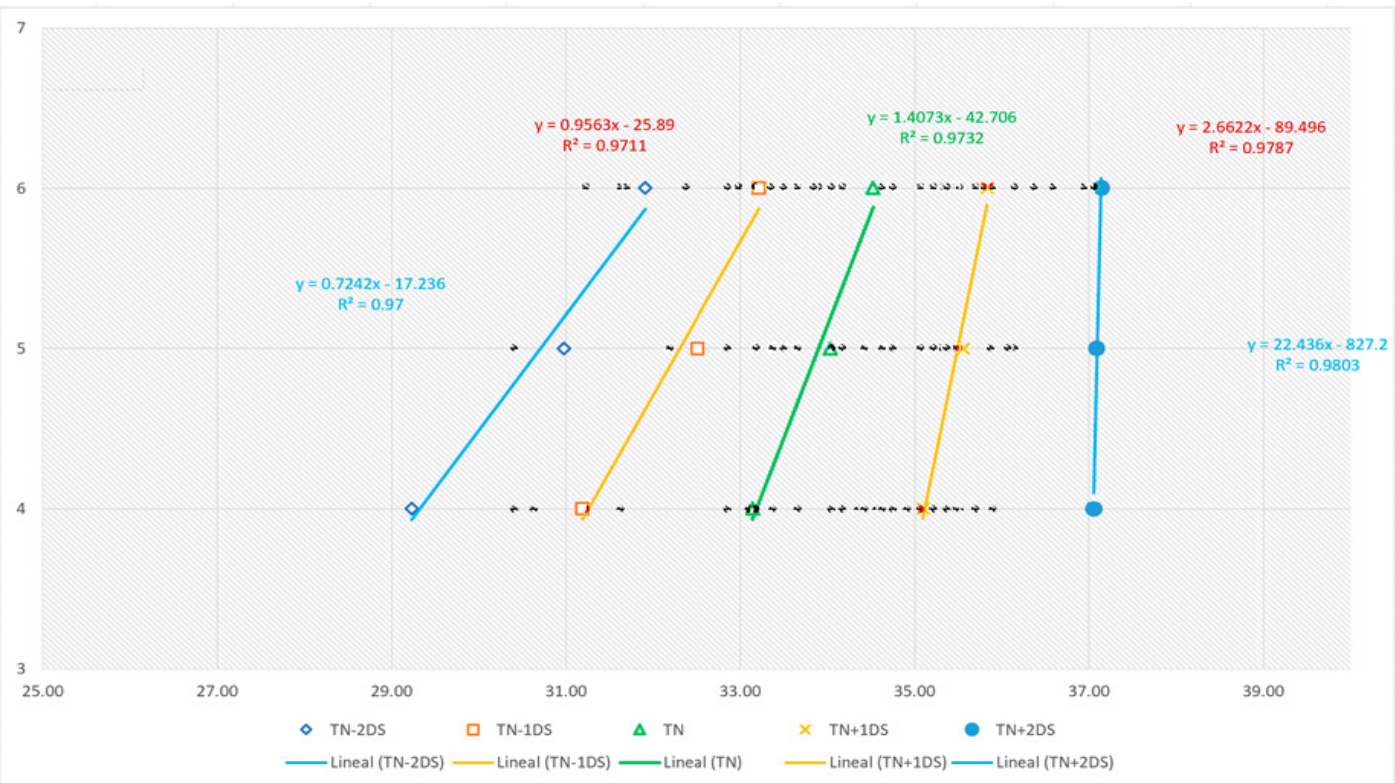

Nota. En la figura se puede apreciar como la mayoría de los votos están dentro del rango reducido y rango extendido

Las diversas variables medio ambientales afectan la sensación de confort térmico, así como las variables fisiológicas, los aspectos socioculturas y psicológicos del usuario. Durante el diseño del instrumento se tomaron en cuenta todos estos aspectos, los cuales se ven reflejados en los resultados obtenidos, pero se debe de tomar en cuenta que al ser la precepción térmica un proceso personal de cada individuo, los votos de sensación pueden variar entre usuarios, aunque se encuentren 
realizando una misma actividad, o que las variables de su entorno sean las mismas. (Guzmán, y Ochoa, 2014)

Para los datos de los resultados recabados en la presente investigación se pudo apreciar que $1.9^{\circ} \mathrm{C}$ resultaron como el rango reducido de sanción térmica para el espacio analizado, y que la Temperatura de neutralidad de el espacio se estableció en $33.14^{\circ} \mathrm{C}$ (Tabla 3)

Tabla 3. Temperatura de neutralidad y variación estándar

\begin{tabular}{lllllllll}
\hline DS & $\begin{array}{l}\text { Sensación } \\
\text { Térmica }\end{array}$ & Escala & TN-2DS & TN-1DS & TN & TN+1DS & TN+2DS \\
\hline 1.96 & $\begin{array}{l}\text { Ni frio } \\
\text { calor }\end{array}$ & ni & 4 & 29.23 & 31.18 & 33.14 & 35.10 & 37.05 \\
\hline 1.53 & $\begin{array}{l}\text { Algo } \\
\text { calor }\end{array}$ & de & 5 & 30.98 & 32.50 & 34.03 & 35.56 & 37.09 \\
\hline 1.31 & Calor & 6 & 31.91 & 33.22 & 34.52 & 35.83 & 37.14 \\
\hline
\end{tabular}

Nota. Tn para cada uno de los tres rangos registrados durante la aplicación de las encuestas y sus rangos reducidos y extensos

Como puede ser apreciado en la tabla anterior las dispersiones estándar van disminuyendo según va subiendo la temperatura media de cada uno de los rangos lo que puede llevar a deducir que entre más alta es la temperatura medio del espacio hay mayor consenso de malestar por sensación térmica en el lugar y entre más se acerca a la temperatura de neutralidad o de confort térmico hay mayor dispersión en los votos de sensación térmica por las diferencias entre los metabolismos y actividades de cada individuo.

\section{Conclusiones y recomendaciones}

Durante la ampliación del instrumento utilizado para valorar el voto de sensación térmica del encuestado, el clima presenta un comportamiento asimétrico con una tendencia a las temperaturas cálidas, por lo que en el cuestionario no se dieron votos por sensaciones frías mostrando una tendencia a la adaptación al periodo cálido de los usuarios.

Si se toman en cuenta la comparación de la Tn la cual fue de $33.14^{\circ} \mathrm{C}$, en contra de la temperatura ambiente máxima registrada en los tres rangos registrados la cual fue de $37.02^{\circ} \mathrm{C}$, se puede apreciar una diferencia de +3.8 , y ya que el rango reducido tubo un valor $\mathrm{de} \pm 1.9^{\circ} \mathrm{C}$, se llega a la conclusión de que es por esta razón que los votos de sensación térmica tienden a tener un valor a la incomodidad térmica, durante la aplicación del instrumento.

Ya que las líneas de la gráfica de regresiones lineales no son paralelas significa que la adaptación de los usuarios al espacio no es simétrica según cambia su percepción de la temperatura de calor a frio, ya que durante los periodos cálidos se tiende a las sensaciones fuera del confort térmico durante la utilización de los espacios exteriores.

Cuando se comparan las diversas variables de cada individuo encuestado, comparadas con las variables del clima y el voto dado a la sensación térmica percibida durante la encuesta, se puede saber cuál fue el nivel de confort en el que se encontraba el encuestado, dando como resultado una temperatura de neutralidad, la cual se puede convertir en una temperatura de diseño para futuros trabajos tomando en cuenta que las áreas sin radiación directa obtuvieron un voto con tendencia favorable a la sensación térmica percibida, se puede utilizar estos datos para lograr alargar el tiempo 
de uso de los espacios por el usuario en el clima seco semiárido.

Tomando en cuenta lo anterior la presente investigación sirve para decidir como mejorar los espacios abiertos exteriores, o como diseñar un nuevo espacio exterior en clima seco semidesértico, ya que con el pueden ser estimadas las condiciones de la temperatura de neutralidad o confort térmico, durante la utilización de los espacios en la zona durante el verano.

\section{Agradecimientos}

A los alumnos de ayudanticas de investigación y becarios, por el trabajo que realizaron en campo, en la captura y procesamiento de datos. A los directivos de FCITEC por el apoyo durante la realización de la investigación. A la Universidad Autónoma de Baja California por el apoyo económico para la realización de esta investigación.

\section{Referencias}

Bojórquez G., Gómez-Azpeitia L., García-Cueto O., Ruiz-Torres R., Luna A., (2010). Temperatura neutral y rangos de confort térmico para exteriores, período cálido en clima cálido seco, Ambiente construido. 10 (2), 133-143. https://doi.org/10.1590/S1678-86212010000200009

Chávez, F. (2002). Zona variable de confort térmico [Tesis Doctoral]. Universitat Politecnica de Catalunya.

Gómez N., Rojas A., Higueras E. (2010). Parámetros Sostenibles en el Planeamiento y Diseño Ambiental del Espacio Micro Urbano. Pluris, 4, 407

Givoni, B., Noguchi, M., Saaroni, H., Pochter, O., Yaacov, Y., Feller, N., Becher, S. (2003). Outdoor comfort research issues. Energy and buildings, 35, 77-86. https://doi.org/10.1016/ S0378-7788(02)00082-8

Guzmán, F., \& Ochoa, J., (2014). Confort térmico en los espacios públicos urbanos clima cálido y frío semiseco. Hábitat sustentable, 4 (2), 52-63. Recuperado a partir de http://revistas.ubiobio.cl/index.php/RHS/article/view/450

International organization for standardization (2005). ISO 7730:2005, Ergonomics of the thermal environment - analytical determination and interpretation of thermal comfort using calculation of the PMV and PPD indices and local thermal comfort criteria.

Nikolopoulou, M., (2004). Designing Open Spaces in the Urban Environment: A Bioclimatic Approach. Rediscovering the Urban Realm and Open Spaces, CRES

Linacre, E., \& Geerts, B. (1997). Climates and Weather Explained. Routledge

Ochoa, J., (2009). Ciudad, vegetación e impacto climático. El confort en los espacios urbanos. Erasmus Ediciones.

Olgyay, V., (2019). Arquitectura y Clima. Manual de Diseño Bioclimático para Arquitectos y Urbanistas. Mc Grill Hill. 